ARTIGO

\title{
SUBTESTE DE ARITMÉTICA: DESEMPENHO DE ESTUDANTES COM INDÍCIOS DE DISCALCULIA DO DESENVOLVIMENTO
}

\author{
ARITHMETIC SUBTEST: STUDENT PERFORMANCE WITH INDICATIONS OF \\ DISCALCULIA OF DEVELOPMENT
}

\begin{abstract}
SUBTEST ARITMÉTICO: RENDIMIENTO ESTUDIANTIL CON INDICACIONES DE DISCALCULIA DE DESARROLLO
\end{abstract}

\author{
Lanúzia Almeida Brum Avila \\ Pontifícia Universidade Católica do Rio Grande do Sul - Brasil \\ Isabel Cristina Machado de Lara \\ Pontifícia Universidade Católica do Rio Grande do Sul - Brasil
}

\begin{abstract}
Resumo: Este artigo apresenta parte dos resultados advindos de uma pesquisa de Mestrado em Educação em Ciências e Matemática sobre Discalculia do Desenvolvimento, em específico, acerca do desempenho de estudantes com indícios desse transtorno no Subteste de Aritmética (STEIN, 1994), o qual foi utilizado como um dos instrumentos de avaliação psicopedagógica. O objetivo é analisar a evolução do desenvolvimento das habilidades matemáticas desses estudantes, após a realização de intervenções psicopedagógicas. A pesquisa aconteceu em três momentos: no primeiro, 11 estudantes com idades entre 9 e 12 anos, participaram da avaliação psicopedagógica, entre eles, o Subteste de Aritmética; em seguida receberam dez sessões de intervenção; por fim, foram reavaliados. A análise dos resultados obtidos baseou-se no escore de correções do teste. No intuito de verificar os avanços no desempenho dos estudantes, apresenta-se uma análise quali-quantitativa. Em relação à análise dos dados, o desempenho dos estudantes, optou-se pela Análise de Conteúdo - AC, considerando as cinco etapas definidas por Moraes (1999): preparação; unitarização; categorização; descrição; e interpretação. A partir da análise das unidades de significado, emergiram 12 categorias iniciais, as quais foram reagrupadas em uma categoria final: Discalculia do Desenvolvimento - DD. Após o período de intervenções psicopedagógicas, baseadas na utilização de jogos específicos de acordo com as habilidades que estavam em defasagem, verifica-se que os estudantes apresentaram avanços na reavaliação, em relação às operações de adição, subtração, multiplicação e divisão.
\end{abstract}

Palavras chave: Discalculia do Desenvolvimento; Habilidades matemáticas; Intervenções psicopedagógicas.

Abstract: This article presents part of the results from a Master's research in Science and Mathematics Education on Developmental Dyscalculia, in particular, about the performance of students with signs of this disorder in the Arithmetic Subtest (STEIN, 1994), which was used as one of the instruments of psychopedagogical assessment. The objective is to analyze the evolution of the development of the mathematical skills of these students, after carrying out psychopedagogical interventions. The research 
took place in three moments: in the first, 11 students aged between 9 and 12 years old participated in the psychopedagogical assessment, among them, the Arithmetic Subtest; then they received ten intervention sessions; finally, they were reassessed. The analysis of the results obtained was based on the test correction score. In order to verify advances in student performance, a qualitative and quantitative analysis is presented. In relation to data analysis, student performance, Content Analysis AC was chosen, considering the five steps defined by Moraes (1999): preparation; unitarization; categorization; description; and interpretation. From the analysis of the units of meaning, 12 initial categories emerged, which were grouped into a final category: Developmental Dyscalculia - DD. After the period of psychopedagogical interventions, based on the use of specific games according to the skills that were out of step, it appears that the students showed advances in reassessment, in relation to the operations of addition, subtraction, multiplication and division.

Keywords: Developmental Dyscalculia; Mathematical skills; Psychopedagogical interventions.

Resumen: Este artículo presenta parte de los resultados de una investigación de maestría en Ciencias y Educación Matemática sobre Discalculia del Desarrollo, en particular, sobre el desempeño de los estudiantes con signos de este trastorno en la Subprueba Aritmética (STEIN, 1994), que se utilizó como Uno de los instrumentos de evaluación psicopedagógica. El objetivo es analizar la evolución del desarrollo de las habilidades matemáticas de estos estudiantes, luego de realizar intervenciones psicopedagógicas. La investigación se llevó a cabo en tres momentos: en el primero, 11 estudiantes de entre 9 y 12 años participaron en la evaluación psicopedagógica, entre ellos, el Subtest de Aritmética; luego recibieron diez sesiones de intervención; finalmente, fueron reevaluados. El análisis de los resultados obtenidos se basó en la puntuación de corrección de la prueba. Para verificar los avances en el desempeño de los estudiantes, se presenta un análisis cualitativo y cuantitativo. En relación con el análisis de datos, el rendimiento de los estudiantes, se eligió el Análisis de Contenido - AC, considerando los cinco pasos definidos por Moraes (1999): preparación; unitarización categorización descripción; e interpretación. Del análisis de las unidades de significado, surgieron 12 categorías iniciales, que se agruparon en una categoría final: Discalculia del Desarrollo - DD. Después del período de intervenciones psicopedagógicas, basadas en el uso de juegos específicos de acuerdo con las habilidades que estaban fuera de lugar, parece que los estudiantes mostraron avances en la reevaluación, en relación con las operaciones de suma, resta, multiplicación y división.

Palabras clave: Discalculia del Desarrollo; Habilidades matemáticas; Intervenciones psicopedagógicas.

\section{Introdução}

Em 2015, constituiu-se o Grupo de Pesquisas e Estudos sobre Discalculia da Pontifícia Universidade Católica do Rio Grande do Sul - GEPEDPUCRS, com o objetivo de desenvolver diferentes estudos e pesquisas acerca do Transtorno de Aprendizagem em Matemática - TAM, embasando-se teoricamente na perspectiva de Kosc (1974), no intuito de compreender o desenvolvimento das habilidades matemáticas as quais estão em defasagens, priorizando os subtipos de Discalculia do Desenvolvimento - DD propostas pelo autor.

A DD foi defina por Kosc (1970 apud KOSC, 1974) como sendo:

Developmental dyscalculia is a structural disorder of mathematical abilities which has its origin in a genetic or congenital disorder of those parts of the 
brain that are the direct anatomico-physiological substrate of the maturation of the mathematical abilities adequate to age, without a simultaneous disorder of general mental functions. ${ }^{1}$ (KOSC, 1970, p. 192 apud KOSC, 1974, p. 165).

Kosc (1974) classificou a DD em seis subtipos, na intenção de denominar e classificar as defasagens relacionadas às habilidades matemáticas presentes nesse transtorno sejam elas: Discalculia verbal; Discalculia practognóstica; Discalculia léxica; Discalculia gráfica; Discalculia ideognóstica; Discalculia operacional.

A Discalculia verbal é definida por Kosc (1974) como estando relacionada às habilidades verbais. Estudantes que apresentam defasagens nessa área tendem a apresentar dificuldade na nomeação de quantidades e numerais e no reconhecimento dos símbolos matemáticos. Em casos mais graves, há incapacidade de identificar números ditados, todavia conseguem ler ou escrever o numeral.

Kosc (1974) refere-se à Discalculia practognóstica, envolvendo dificuldades na manipulação de objetos ou figuras. O estudante apresenta dificuldade, por exemplo, para organizar bastões considerando a magnitude dos mesmos. Em casos de dificuldades acentuadas, até mesmo mostrar qual bastão é maior ou menor, torna-se um grande desafio.

Em se tratando da Discalculia léxica, segundo o autor (1974) as dificuldades estão relacionadas à leitura dos símbolos matemáticos na resolução de cálculos, sendo comum a troca de sinais.

Em relação à Discalculia gráfica, a dificuldade refere-se à escrita dos símbolos matemáticos. Um estudante com esse subtipo de DD pode ter comorbidade com Disgrafia e Dislexia (KOSC, 1974).

Estudantes com Discalculia ideognóstica, de acordo com o autor apresentam dificuldades na compreensão de conceitos matemáticos e na resolução de operações mentais. Para alguns estudantes, até mesmo resolver somas simples, é uma grande dificuldade. Kosc $(1974$, p. 168) se reporta a um exemplo destacando que um estudante pode "[...] knows that 9 = "nine" and that "nine" is to be written as 9, but he does not know that 9 or nine is one less than 10, or $3 \times 3$, or one-half of 18 , etc.". 2

\footnotetext{
${ }^{1}$ Discalculia do Desenvolvimento é uma disfunção estrutural de habilidades matemáticas, que tem sua origem numa deficiência genética ou congênita dessas partes do cérebro, que são os substratos anatômico-fisiológicos diretos da maturação das habilidades matemáticas de acordo com a idade, sem uma disfunção simultânea de funções mentais gerais (tradução nossa).

${ }^{2}[\ldots]$ sabe que 9 = "nove" e que "nove" é escrito como "9", mas ela não sabe que 9 ou nove é 10 menos um, ou 3 x 3 , ou metade de 18 , etc. (tradução nossa).
} 
Referente à Discalculia operacional, os estudantes apresentam dificuldades na resolução de operações, ocorrendo à troca de operações, como por exemplo, em um cálculo de adição realizar uma subtração, ou vice-versa (KOSC, 1974).

Preocupando-se com esse tema, desenvolveu-se a pesquisa "Avaliação e intervenções psicopedagógicas em crianças com indícios de Discalculia” (AVILA, 2017). Neste artigo apresenta-se um recorte dessa pesquisa, destacando os resultados advindos do Subteste de Aritmética (STEIN, 1994), instrumento utilizado como uma das etapas de avaliação psicopedagógica. O Subteste foi aplicado com 11 estudantes com idades entre 9 e 12 anos e reaplicado na reavaliação, após as dez sessões de intervenção psicopedagógica. Delimita-se, nesse artigo, à apresentação dos resultados obtidos com os estudantes participantes da pesquisa. Teve-se como objetivo: “Analisar a evolução das habilidades matemáticas envolvidas na Discalculia em estudantes com indícios desse transtorno, após a realização de intervenções psicopedagógicas".

Com base no objetivo proposto, adotou-se a Análise de Conteúdo - AC, baseando-se em Moraes (1999) que propõe cinco etapas para a realização da análise: preparação; unitarização; categorização; descrição; interpretação. Considerando a análise das unidades de significado, emergiram 12 categorias iniciais, seis categorias intermediárias das quais originouse uma categoria final: Discalculia do Desenvolvimento.

\section{Dificuldades e Transtornos de Aprendizagem}

As Dificuldades de Aprendizagem - DA - podem estar relacionadas a problemas passageiros, por exemplo, o conteúdo escolar, separação dos pais, perda de alguém e falta de motivação, ou podem ser decorrentes de situações secundárias, como alterações das funções sensoriais, doenças crônicas, transtornos psiquiátricos e doenças neurológicas (RELVAS, 2015).

De acordo com Fonseca (2000, p. 98),

[...] se torna difícil determinar a natureza precisa das causas endógenas das DA. Envolvimentos familiares pobres, relação criança-adulto distorcidas, expectativas negativas, erros pedagógicos [...] situações de aprendizagem limitativa, $[\ldots]$ podem também produzir DA.

Todavia, alguns fatores podem estar influenciando negativamente para que a aprendizagem deixe de acontecer, de acordo com Relvas (2015) pode-se destacar como causas a escola, a família e ou o próprio estudante. Em relação aos fatores relacionados à escola, a 
autora (2015) menciona que as condições físicas do espaço escolar, as condições pedagógicas e as condições do corpo docente, são primordiais para que ocorra a aprendizagem, podendo assim ser uma causa de DA. Referente à família, a autora (2015) destaca que a escolaridade dos pais, o hábito de leitura e problemas familiares são fatores que afetam o processo de aprendizagem. Questões relacionadas a comprometimentos físicos, psicológicos e neurológicos, também podem ocasionar DA (RELVAS, 2015).

Na mesma perspectiva, Ohlweiler (2006, p. 127) ressalta que:

As dificuldades de aprendizagem podem ser [...] de percurso, causadas por problemas da escola e/ou da família, que nem sempre oferecem condições adequadas para o sucesso da criança [...] dificuldades que a criança pode apresentar em alguma matéria ou em algum momento da vida, além de problemas psicológicos, como falta de motivação e baixa auto-estima.".

Em específico sobre as Dificuldades de Aprendizagem em Matemática - DAM - em algumas situações, são possíveis de serem identificadas desde a Educação Infantil, em que inicia-se o processo de construção do número (AVILA; LARA, 2017). Entretanto, as autoras (2017) destacam que por vezes torna-se difícil o professor sozinho identificar se as DAM, pelas quais os estudantes estão passando, estão relacionadas a um conteúdo específico ou a uma única disciplina. "Em alguns casos, se faz necessário o auxílio do setor pedagógico da escola, almejando a busca de informações sobre a história de vida desse estudante, por meio de uma conversa com os pais." (AVILA; LARA, 2017, p. 37).

Em se tratando das DAM, Vieira (2004, p. 110) especifica que:

Muitas vezes, o aluno enfrenta [...] DAM, em razão de lhe "ensinarem" através da memória mecânica, sem nunca estimularem a aprendizagem a partir da assimilação dos conceitos básicos, tais como classificação, ordenação, sequência e ordenação, correspondência, conservação.

O modo como o professor ministra suas aulas, segundo Lara (2004, p. 141) pode ser um dos fatores das DAM. Conforme a autora,

[...] o modo como os saberes matemáticos vêm sendo tratados fazem com que a Matemática passe a ser um corpo fechado de saberes verdadeiros, atemporais e irrefutáveis, passível então, de transmissão [...] o aluno constitui-se como um mero receptor e repetidor [...] a maneira como a Matemática aparece em alguns livros didáticos reflete a imagem de um conjunto de conteúdos ou definições hierarquizadas e entrelaçadas).

Compreender como ocorre o processo de aprendizagem é uma tarefa difícil, pois cada estudante é único e aprende de um modo específico. Deve-se considerar ainda que cada estudante possui conhecimentos prévios diferenciados, os quais trazem consigo de acordo com 
o contexto cultural no qual estão imersos. Faz-se necessário o professor "[...] compreender como evolui o pensamento e interpretar comportamentos não é trabalho apenas de psicólogos ou psicopedagogos, mas também do professor.” (LARA, 2004, p. 145).

Assim sendo, percebe-se que as DA podem ocorrer com muitos estudantes ao longo da fase escolar, em determinada tarefa, em determinada disciplina, por um período, o que não necessariamente significa um Transtorno de Aprendizagem - TA, que ocasiona problemas acentuados na aprendizagem (OHLWEILER, 2006).

De acordo com o DSM-5:

Um Transtorno Mental é uma Síndrome caracterizada por perturbação clinicamente significativa na cognição, na regulação emocional ou no comportamento de um indivíduo que reflete uma disfunção nos processos psicológicos, biológicos ou de desenvolvimento subjacentes ao funcionamento mental (2014, p. 20).

O TA compreende uma inabilidade específica, acentuando problemas na leitura, escrita e/ou matemática, em que os estudantes apresentam desempenho abaixo do esperado, se comparado com outros estudantes da mesma faixa etária e escolaridade (RELVAS, 2015). Pode-se suspeitar de TA em estudantes que apresentem: "[...] inteligência normal; ausência de alterações motoras ou sensoriais; bom ajuste emocional, porém "camuflado"; nível socioeconômico e cultural aceitável." (RELVAS, 2015, p. 53).

De acordo com a CID-10, o TA:

[...] são transtornos nos quais os padrões normais de aquisição de habilidades são perturbados desde os estágios iniciais do desenvolvimento. Eles não são simplesmente uma consequência de uma falta de oportunidade de aprender nem são decorrentes de qualquer forma de traumatismo ou de doença cerebral adquirida. Ao contrário, pensa-se que os transtornos originam-se de anormalidades no processo cognitivo, que derivam em grande parte de algum tipo de disfunção biológica. (1993, p. 236).

Os TA são denominados por Ohlweiler (2006) como sendo transtornos específicos, os quais afetam significativamente as habilidades escolares, não sendo resultantes de outros transtornos, todavia podem ter comorbidades. Contudo, para o diagnóstico de TA alguns aspectos devem ser considerados, segundo Ohlweiler (2006, p. 128): “[...] deve estar presente desde os primeiros anos de vida [...]"; mesmo com tratamento especializado, irão persistir os problemas, por vezes por toda vida; o comprometimento deve ser significativamente abaixo do esperado (OHLWEILER, 2006).

As DA e TA sempre existiram, porém o que se modificou segundo Moojen e Costa (2006) foi à visão dos pais sobre os problemas que afetam a aprendizagem. "O problema de 
aprendizagem, pela sua magnitude e importância nos dias atuais, requer a interação entre os diversos profissionais que lidam com o caso, particularmente para o estabelecimento do diagnóstico [...]" (MOOJEN; COSTA, 2006, p. 111). Com base nos aspectos mencionados, convém salientar a necessidade do profissional que está realizando a avaliação compreenda as causas das dificuldades pelas quais o estudante está vivenciando, de modo a realizar um diagnóstico correto (MOOJEN; COSTA, 2006).

\section{Procedimentos metodológicos}

Com o objetivo de "Analisar a evolução das habilidades matemáticas envolvidas na Discalculia de estudantes com indícios desse transtorno, após a realização de intervenções psicopedagógicas", optou-se na pesquisa, a qual originou o respectivo artigo o método misto de pesquisa, adotando aspectos tanto da pesquisa quantitativa, como da qualitativa. Isso justifica-se pela intenção de analisar qualitativamente os resultados de cada estudante nos testes.

Com o intento de possibilitar aos participantes da pesquisa obterem avanços em relação as habilidades matemáticas em defasagem, optou-se por adotar como delineamento de pesquisa, a pesquisa experimental, por ser frequentemente utilizada nas ciências humanas, em específico acerca de estudos envolvendo aprendizagem em pequenos grupos de sujeitos (GIL, 2002).

\section{Instrumento para coleta de dados}

Na intenção de produzir os dados necessários neste estudo, utilizou-se para a coleta de dados: anamnese com os responsáveis; Escala Wechsler Abreviada de Inteligência - WASI (2014); Subteste de Aritmética; Intervenções psicopedagógicas; Reavaliação psicopedagógica.

\section{Subteste de Aritmética (STEIN, 1994)}

O instrumento designado como Subteste de Aritmética, é composto de cálculos aritméticos com grau de dificuldade crescente, correspondentes aos conteúdos desenvolvidos da $1^{\text {a }}$ a $6^{\mathrm{a}}$ séries $\left(2^{\mathrm{o}}\right.$ ao $7^{\mathrm{o}}$ anos) do Ensino Fundamental, vistos como alicerces de conteúdos estudados posteriormente.

Iniciou-se explicando que o Subteste de Aritmética foi elaborado para estudantes de todas as idades, por este motivo as questões iniciais são bem simples e gradativamente vai aumentando o seu grau de complexidade ao longo do mesmo. Assim, ressaltou-se que o 
estudante deveria resolver as questões que apresentassem conceitos que já haviam sido desenvolvidos em sala de aula ou que ela tivesse conhecimento prévio. Aplicou-se com todos os estudantes os três itens da parte oral, anotando as respectivas respostas dadas ao lado de cada uma das questões no protocolo. Entregou-se, o protocolo do Subteste e solicitou-se que resolvesse as operações, como já foi combinando em um primeiro momento, mencionando-se que não poderiam fazer uso da borracha na resolução das operações, quando se dessem conta de algum erro, em alguma das operações, devendo, portanto, refazer ao lado, sem apagar. O teste foi reaplicado por três examinadoras com os 11 estudantes selecionadas para participarem das intervenções psicopedagógicas, tendo seus resultados analisados com base na padronização do próprio teste.

\section{Seleção dos participantes da pesquisa}

O respectivo estudo é um fragmento dos resultados da pesquisa "Avaliação $e$ intervenções psicopedagógicas em crianças com indícios de Discalculia” (AVILA, 2017). A pesquisa foi analisada e obteve aprovação do comitê de ética em pesquisa.

A amostra da pesquisa que originou este artigo é composta por 11 estudantes, na faixa etária entre 9 anos e 12 anos e 11 meses, do Ensino Fundamental. Com a intenção de preservar o anonimato, cada participante é denominado pela expressão "E1, E2...”.

Os dados da amostra foram adquiridos de diferentes modos: da avaliação pelo projeto Avaliação de Crianças em Risco de Transtornos de Aprendizagem - ACERTA; da busca ao banco de dados do projeto ACERTA; ou da triagem psicopedagógica com estudantes de duas escolas públicas de Capivari do Sul, cidade do estado do Rio Grande do Sul.

Referente à avaliação pelo projeto ACERTA, os responsáveis por seis estudantes, realizaram o processo de avaliação de seus filhos no ambulatório do Hospital São Lucas da PUCRS. Perfazendo as seguintes etapas: em um primeiro momento, os responsáveis interessados em seus filhos participarem do projeto, realizaram o encaminhamento de e-mail para projetoacerta@gmail.com. Posteriormente, um dos participantes do projeto, entrou em contato com os familiares, ressaltando os critérios para participar da pesquisa, agendando dia e horário para realização da anamnese com os responsáveis e realização da triagem com o estudante, referente à leitura, escrita e avaliação da Matemática. Em outro momento, os participantes foram convidados a submeter-se a Escala Wechsler Abreviada de Inteligência WASI (2014), aplicado pelas psicólogas da equipe do projeto. Dando continuidade ao processo de avaliação multidisciplinar, os estudantes passaram pela avaliação com a fonoaudióloga. Para 
finalizar o processo, foi realizada a devolução do diagnóstico referente ao processo de leitura e escrita.

Adicionado a isso, buscou-se no banco de dados do projeto ACERTA, estudantes que apresentassem queixa de DAM, as quais foram salientadas na anamnese pelos responsáveis. A partir dessa busca, selecionou-se 17 estudantes. Primeiramente, realizou-se o contato telefônico explicando os critérios necessários para ingressar na pesquisa e agendou-se com os interessados uma nova anamnese, porém com detalhes sobre as DAM. No dia da entrevista de anamnese, a psicopedagoga agendou a avaliação de Matemática, a qual priorizou a utilização do Teste de Transcodificação (MOURA et al., 2013) e do Subteste de Aritmética (STEIN, 1994).

Além das coletas de dados mencionadas anteriormente, foram selecionadas duas escolas públicas de Capivari do Sul, para realizar a avaliação psicopedagógica com estudantes com DAM e indícios de DD. A avaliação desenvolveu-se do mesmo modo que a anterior, porém após os testes, foi agendado com as famílias a Escala Wechsler Abreviada de Inteligência WASI (2014), no ambulatório do hospital São Lucas da PUCRS.

Convém salientar, que os estudantes e seus respectivos responsáveis, assinaram o Termo de Assentimento e o Termo de Consentimento Livre e Esclarecido.

Os estudantes selecionados para participar das intervenções psicopedagógicas, foram organizados em dois grupos, um grupo denominado controle, com seis estudantes e um grupo experimental, composto por nove estudantes. Sete estudantes do grupo experimental concluíram o processo de intervenção, pois um estudante desse grupo apresentava transtorno psiquiátrico grave, o que foi omitido pela mãe na anamnese e outro estudante por motivos particulares não tinha possibilidade de comparecer aos atendimentos.

As intervenções com o grupo controle ocorreram no município de Capivari do Sul, nos meses de outubro e novembro de 2016. Foram desenvolvidas cinco aulas, totalizando assim 10h de intervenções em grupo. Os estudantes que permaneceram com indícios de DD, foram convidados a participarem de cinco sessões individuais.

Os estudantes do grupo experimental participaram de dez sessões individuais de intervenções psicopedagógicas com 1h de duração cada uma durante os meses de setembro a novembro de 2016.

\section{Técnicas de análise}

Como mencionado anteriormente, para realizar a avaliação do desempenho de cada estudante utilizou-se o Subteste de Aritmética (STEIN, 1994). Para verificar e corrigir esses resultados seguiu-se o indicado pela literatura do teste. 
No que se refere à análise dos dados do Subteste de Aritmética (STEIN, 1994) é realizado computando-se os acertos, considerando que cada item correto vale um ponto. A soma dos pontos do Subteste, de acordo com Stein (1994) é chamada Escore Bruto (EB). Para avaliar o desempenho de cada estudante foi utilizada a Tabela de Normas que apresenta os escores brutos por série escolar.

Vale ressaltar que as seis tabelas apresentadas por Stein (1994) correspondem às fases escolares de $1^{\text {a }}$ série à $6^{\mathrm{a}}$ série, consideradas neste estudo como sendo do $2^{\circ}$ ao $7^{\circ}$ ano, respectivamente. Além disso, foi utilizado o Crivo para correção do Subteste de Aritmética.

Com o propósito de analisar a evolução do desenvolvimento das habilidades matemáticas, após a realização de intervenções psicopedagógicas, utilizou-se como análise dos dados a AC, considerando as cinco etapas definidas por Moraes (1999). A AC inicia-se pela preparação das informações, selecionando os materiais relacionados aos objetivos da pesquisa e posteriormente, codificam-se esses materiais, na intenção de identificar os materiais a serem analisados. Após, realiza-se a unitarização, etapa em que o pesquisador irá reler atentamente os materiais a serem analisados, definindo a unidade de análise, a qual será posteriormente classificada. Em seguida; o pesquisador dedica-se a categorização, agrupando os dados considerando as semelhanças entre os dados. Posteriormente, com as categorias definidas, é o momento de comunicar os resultados advindos do material de análise, dedicando-se a etapa de descrição. Para finalizar a AC, o pesquisador remete-se a desenvolver a etapa de interpretação, buscando estabelecer a compreensão e interpretação.

\section{Discussão dos resultados}

Com base na análise dos resultados dos estudantes no pré-teste e pós-teste e a partir da análise das unidades de significado constituídas pelas resoluções apresentadas por esses estudantes, buscaram-se por semelhanças que deram origem a 12 categorias iniciais, as quais foram reagrupadas em seis categorias intermediárias.

Para realizar a análise das resoluções ou respostas dadas pelos estudantes, levaram-se em conta as habilidades envolvidas em cada um dos subtipos de DD definidos por Kosc (1974). Contudo, foi possível verificar que na maioria dos casos as habilidades em defasagem, apresentadas pelos estudantes, correspondiam a mais de um subtipo de DD. Diante disso, o Quadro 1, apresenta as categorias intermediárias e a categoria final emergente. 
Quadro 1: Categorias intermediárias e categoria final

\begin{tabular}{|c|c|}
\hline Categorias Intermediárias & Categoria Final \\
\hline Discalculia ideognóstica e Discalculia operacional & \multirow{9}{*}{ Discalculia do Desenvolvimento } \\
\hline Discalculia léxica, Discalculia ideognóstica, e & \\
\hline Discalculia operacional & \\
\hline Discalculia verbal, Discalculia practognóstica, & \\
\hline Discalculia léxica, Discalculia gráfica, Discalculia & \\
\hline ideognóstica e Discalculia operacional & \\
\hline $\begin{array}{c}\text { Discalculia verbal, Discalculia gráfica, Discalculia } \\
\text { ideognóstica e Discalculia operacional }\end{array}$ & \\
\hline Discalculia ideognóstica & \\
\hline $\begin{array}{c}\text { Discalculia verbal, Discalculia ideognóstica, } \\
\text { Discalculia operacional }\end{array}$ & \\
\hline
\end{tabular}

Fonte: Elaborado pelas autoras

Com base nessas categorias produziram-se os textos-síntese de modo descritivo e interpretativo, relacionando os resultados das avaliações com as ideias defendidas por Haase; Costa; Michelli; Oliveira; Wood (2011, p. 143), Haase; Moura; Chagas, Wood (2011), Kosc (1974) buscando a compreensão e interpretação, implícita no corpus de análise. Destaca-se que as avaliações dos estudantes são apresentadas em itálico para diferenciar das citações dos autores. Os estudantes são denominados pela expressão “E1, E2...”, seguido de um número que as individualiza para garantir o anonimato.

\section{Discalculia do Desenvolvimento}

Uma análise detalhada do desempenho dos estudantes possibilitou verificar que em relação ao Subteste de Aritmética (STEIN, 1994), os estudantes obtiveram avanços no número de acertos, demonstrando assim, melhoras em seu desempenho. O que é possível verificar, por exemplo, no escore do estudante E3: "Em seu pré-teste obteve oito acertos, tendo como classificação um escore inferior $\leq 20$, e no pós-teste obteve 14 acertos, com um escore inferior $\leq 20$, apresentando assim um avanço de seis acertos." (AVILA, 2017, p. 167-210).

Com o intuito de ilustrar e sintetizar as modificações no desempenho dos estudantes submetidas às intervenções psicopedagógicas, elaborou-se o Gráfico 1. 
Gráfico 1 - Subteste de Aritmética: Pré-teste com Pós-teste

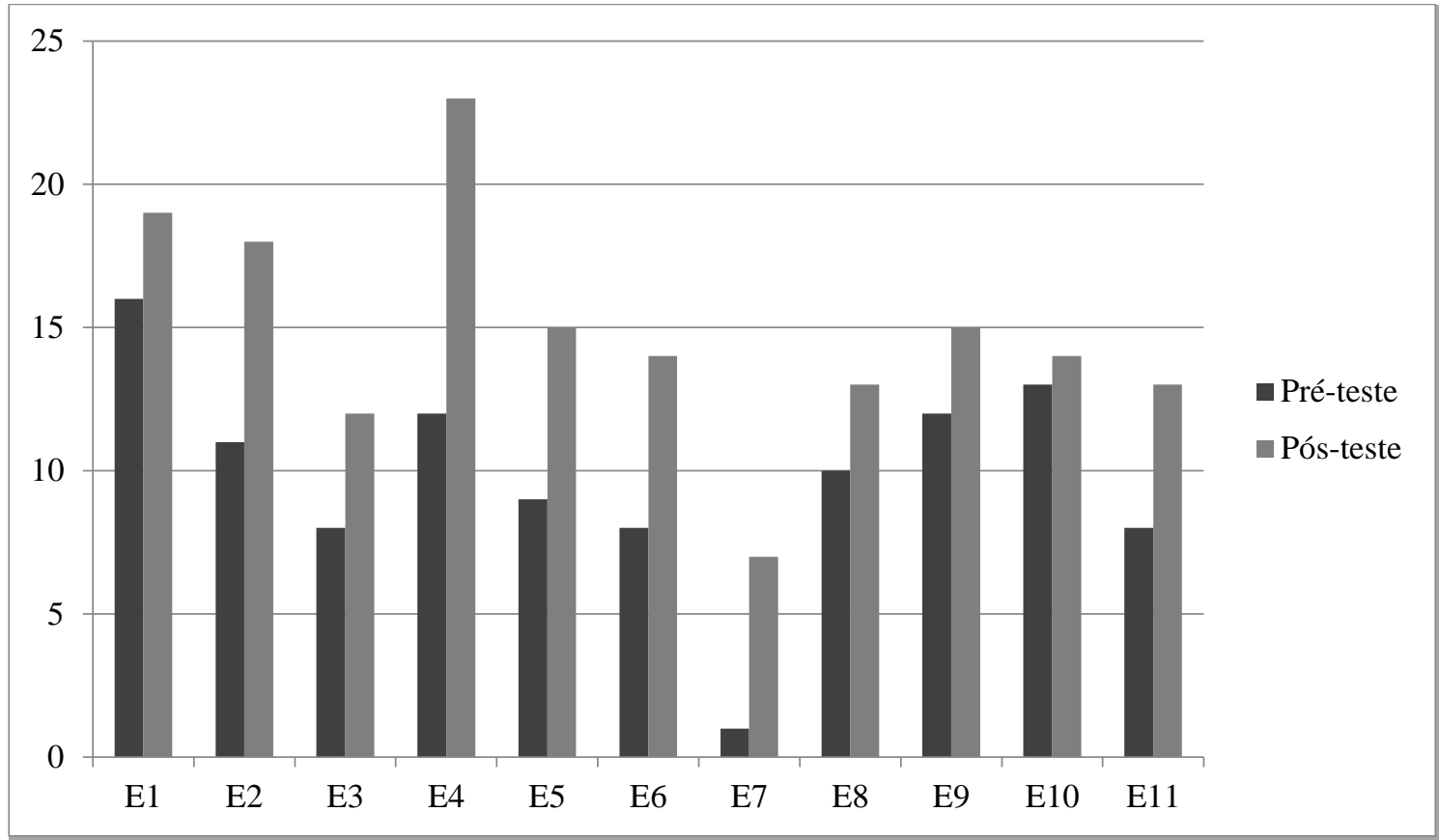

Fonte: Elaborado pelas autoras

Com base no Gráfico 1, é possível perceber que todos os estudantes apresentaram elevação quanto ao número de acertos no pós-teste, apontando que apresentaram melhoras em suas habilidades cognitivas, após as dez sessões de intervenções psicopedagógicas individuais. Desse modo, evidencia que os jogos utilizados nos atendimentos foram eficazes para o tratamento de estudantes com indícios de DD. Dessa forma, pode-se concluir que as habilidades matemáticas que estão em defasagem e as habilidades existentes, podem ser potencializadas.

Referente ao Subteste de Aritmética (STEIN, 1994), observou-se que os estudantes obtiveram avanços no número de acertos do Subteste, demonstrando assim melhoras em seu desempenho. Todavia, mesmo após o período de intervenções, alguns estudantes continuaram apresentando defasagens, principalmente no que concerne a multiplicação e divisão. Um exemplo encontra-se na avaliação do estudante E11.

Em se tratando do Subteste de Aritmética (STEIN, 1994), percebeu-se que a criança teve defasagens quanto às habilidades relacionadas à: [...] multiplicação a partir de dois algarismos no multiplicando e dois algarismos no multiplicador. Foi possível detectar, por meio do subteste, que não sabe realizar operações de divisão. A partir da análise dos dados no escore bruto de Stein (1994), evidenciou-se que a criança obteve 13 acertos, tendo como classificação um escore inferior < 14 (AVILA, 2017, p. 215).

Além disso, em alguns casos não foi possível desenvolver atividades que dessem conta de todas as demandas apresentadas, pois as dificuldades centravam-se desde a construção do número até os conteúdos estudados no respectivo ano escolar, sendo necessário assim, 
proporcionar um número maior de intervenções para esses estudantes, usando-se de outros jogos específicos às habilidades que continuam em defasagens.

Todavia, é perceptível que os jogos desenvolvidos, na maioria dos casos, possibilitaram avanços no desempenho em Matemática, proporcionando que por meio do lúdico, fossem trabalhadas as dificuldades específicas apresentadas por cada um dos estudantes no período de intervenção psicopedagógica.

Contudo, mesmo diante dos avanços, 11 estudantes, os quais contemplam essa categoria, continuaram apresentando defasagens acentuadas, após o período de intervenção psicopedagógica permanecendo com hipótese diagnóstica indícios de DD. Como se pode perceber na avaliação do estudante E5:

No pré-teste teve nove acertos, correspondente a um escore inferior $\leq 20$ e no pós-teste passou para 15 acertos, porém mesmo com avanço de seis acertos, permaneceu com um escore inferior $\leq 20$. [...] estando muito abaixo do esperado para a faixa escolar. Porém, mesmo diante dos avanços, perdurouse como hipótese diagnóstica indícios de Discalculia ideognóstica, pois a resolução de algumas operações mentalmente ainda é um processo difícil para essa criança e, além disso, de Discalculia operacional, pois seu desempenho em relação às operações de adição, subtração, multiplicação e divisão, continua abaixo do esperado para a faixa escolar (AVILA, 2017, p. 209).

As defasagens em relação às habilidades matemáticas permaneceram, mesmo após o período de intervenções, pois segundo Kosc (1970 apud KOSC, 1974, p. 166) "The term "dyscalculia" refers specifically to a disorder of the special abilities for mathematics without a simultaneous defect in general mental abilities. ", sendo necessário assim, que esses estudantes tivessem a oportunidade de continuarem o atendimento psicopedagógico.

Convém destacar que ao longo desse estudo, adotou-se como embasamento teórico as ideias defendidas por Kosc (1974), considerando assim, a classificação de subtipos de DD proposta pelo autor. A seguir apresentam-se as habilidades em prejuízo em cada subtipo, relacionando a hipótese diagnóstica dos participantes da pesquisa. O estudante E4:

[...] apresentou avanços significativos em relação a suas habilidades
matemáticas nas operaçães de adição, subtração, multiplicação, divisão e
frações, estando na média do esperado para a faixa escolar. Evidencia-se que
seus avanços foram decorrentes da potencialização das habilidades
matemáticas existentes e da reabilitação das habilidades matemáticas em
prejuízo. Porém, mesmo diante dos avanços, permaneceu como hipótese
diagnóstica indícios de Discalculia ideognóstica, pois a resolução de algumas

${ }^{3} \mathrm{O}$ termo "discalculia" se refere especificamente a uma disfunção das habilidades especiais para matemática sem um defeito simultâneo nas habilidades mentais gerais (tradução nossa). 
operações mentalmente, ainda é um processo difícil para essa criança (AVILA, 2017, p. 201).

Kosc (1974) reporta-se a Discalculia ideognóstica como sendo uma defasagem na compreensão dos conceitos matemáticos e na realização das operações mentais, o que pode ser percebido até mesmo em somas simples. Um exemplo do grau de dificuldade pode ser verificado, a partir dos dados obtidos na avaliação do estudante E3, em que um dos subtipos de DD observadas após a análise dos testes é a “[...] Discalculia ideognóstica, devido a suas dificuldades acentuadas na compreensão dos conceitos matemáticos, em específico relacionado ao processo da divisão e também, a impossibilidade de realizar operações simples mentalmente." (AVILA, 2017, p. 200).

Além da Discalculia ideognóstica, Kosc (1974) refere-se à outro subtipo de DD, a qual denomina de Discalculia verbal, na qual as dificuldades afetam as habilidades verbais, referente aos termos e símbolos matemáticos, ocasionando dificuldade na nomeação de números e numerais. Conforme Kosc (1974, p. 167):

There are cases of braindamaged persons who are not able to identify the numbers dictated to them in the form of numerals (for example: to show the dictated number of fingers) although they are able to read or write the respective number or to count the amount of things (sensory-verbal dyscalculia). Or, on the other hand, persons with verbal dyscalculia are not able to name the amount of presented things or the value of written numbers although they are able to read or write the dictated number (motor-verbal dyscalculia). ${ }^{4}$

Corroborando as ideias defendidas por Kosc (1974), exemplifica-se com a hipótese diagnóstica de um dos subtipos de DD apresentadas pelo estudante E7: "[...] Discalculia verbal, pelas dificuldades significativas na nomeação e reconhecimento dos numerais e dos números [...]” (AVILA, 2017, p. 211).

Dentre os seis subtipos de DD, destaca-se também a Discalculia practognóstica, em que o estudante tende a apresentar dificuldade para manipular objetos ou figuras, no que tange a enumeração e comparação de quantidades. Logo, o autor (1974, p. 167) expõe que "a patient with practognostic dyscalculia is not able to set out sticks or cubes according to the order of their magnitudes, not even to show which of the two sticks or cubes is bigger or smaller, or whether they are the same size.".

\footnotetext{
${ }^{4}$ Existem casos de pessoas com dano cerebral que não são capazes de identificar números ditados para elas na forma de numerais (por exemplo: de mostrar com o dedo o número ditado) embora sejam capazes de ler ou escrever o respectivo numeral ou contar a quantidade de coisas (Discalculia sensor-verbal) (tradução nossa).

${ }^{5}$ Um paciente com Discalculia practognóstica não é capaz de organizar bastões ou cubos de acordo com sua ordem de magnitude, nem mesmo mostrar qual dos dois cubos ou bastões maiores ou menores, ou se eles são do mesmo tamanho (tradução nossa).
} 
As questões destacadas por Kosc (1974) sobre a Discalculia practognóstica são perceptíveis analisando os resultados da avaliação do estudante E7: “[...] permaneceu como hipótese diagnóstica indícios de [...] Discalculia practognóstica, se considerarmos que está frequentando o $3^{\circ}$ ano e tem defasagens graves na enumeração dos objetos [...]” (AVILA, 2017, p. 211).

A Discalculia léxica é perceptível na hipótese diagnóstica do estudante E6: “[...] se considerarmos que está no $6^{\circ}$ ano e continua apresentando dificuldade na leitura de símbolos matemáticos.” (AVILA, 2017, p. 210). No que se refere à Discalculia léxica, Kosc (1974) menciona que esse subtipo de DD os estudantes apresentam dificuldades na resolução de cálculos matemáticos, pois à leitura dos símbolos matemáticos encontra-se em defasagens quanto aos sinais; dígitos e numerais. Para o autor (1974):

By far the most serious form of lexical dyscalculia is when the child is not able to read the isolated digits and/or simple operational symbols $(+,-, x,-=-$, etc.). In the less serious forms, he cannot read multidigit numbers (especially with more than one zero in the middle), numbers written in a horizontal rather than a vertical line, fractions, squares and roots, decimal numbers, and so on. In some cases he interchanges imilar looking digits ( 3 for 8,6 for 9, and vice versa), or two digit numbers are read as reversed (12 as 21$)^{6}$. (KOSC, 1974, p. 167).

Outro subtipo de DD defendido por Kosc (1974) é a Discalculia gráfica, ocasionando defasagens em relação às habilidades matemáticas, as quais envolvem a escrita de símbolos matemáticos. Estudantes com esse subtipo de DD podem presentar comorbidade com Disgrafia e Dislexia (KOSC, 1974). Essa defasagem verifica-se nos resultados apresentados pelo estudante E8: “[...] Discalculia gráfica, devido ao fato de apresentar erros acentuados na escrita dos numerais [...]” (AVILA, 2017, p. 212).

A Discalculia operacional é definida por Kosc (1974) como afetando as habilidades na resolução de operações, em alguns casos, o estudante pode cometer a troca de operações. Sendo possível verificar, por exemplo, a realização de uma subtração quando o algoritmo era adição, ou vice-versa (KOSC, 1974). De acordo com Kosc (1974):

A typical occurrence is the interchange of operations, e.g., doing addition instead of multiplication; subtraction instead of division; or substitution of more complicated operations by simpler ones (e.g., $12+12=(10+10)+$ $(2+2) ; 3 \times 7=7+7+7=21$; or in serious disturbances: 777$)$. Typical also is a

\footnotetext{
${ }^{6}$ De longe, a forma mais séria de Discalculia léxica é quando a criança não é capaz de ler os dígitos isolados e/ou símbolos operacionais simples $(+,-, \times, \div$, etc). Nas formas menos sérias, ele não consegue ler numerais com muitos dígitos (especialmente com mais de um zero no meio), numerais escritos na horizontal ao invés de na vertical, frações, raízes quadradas, números decimais, e assim em diante. Em alguns casos, ele troca dígitos visualmente parecidos (3 por 8, 6 por 9 e vice-versa), ou dois dígitos numéricos são lidos ao contrário (12 como 21) (tradução nossa).
} 
preference for written calculation of sums (tasks) which could be easily calculated silently, or calculation by counting on the fingers where the task could be easily solved silently or in writing and without counting fingers. ${ }^{7}$ (KOSC, 1974, p. 168).

As defasagens quanto às habilidades na resolução de operações, pode ser evidenciada na avaliação do estudante E2:

[...] defasagens quanto às habilidades relacionadas à: adição de numerais com quatro algarismos e três parcelas; subtração de numerais com três algarismos no minuendo dois algarismos no subtraendo; divisão a partir de dois algarismos no dividendo e um algarismo no divisor. [...] podendo-se ter como hipótese diagnóstica indícios de Discalculia operacional (AVILA, 2017, p. 198).

Com o intuito de ilustrar e quantificar as hipóteses diagnósticas dos estudantes após o período de intervenções psicopedagógicas, elaborou-se o Gráfico 2, tendo como critério para agrupamento o desempenho cognitivo dos estudantes após a reavaliação psicopedagógica.

Gráfico 2 - Hipótese diagnóstica dos estudantes

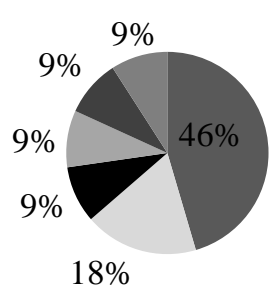

- Discalculia ideognóstica e Discalculia operacional

Discalculia léxica, Discalculia ideognóstica e Discalculia operacional

- Discalculia verbal, Discalculia pragtognóstica, Discalculia léxica, Discalculia gráfica, Discalculia ideognóstica e Discalculia operacional

- Discalculia verbal, Discalculia gráfica, Discalculia ideognóstica e Discalculia operacional

- Discalculia ideognóstica

- Discalculia verbal, Discalculia ideognóstica e Discalculia operacional

Fonte: Elaborado pelas autoras

\footnotetext{
${ }^{7}$ Uma típica ocorrência é a troca das operações, por exemplo, fazer uma adição ao invés de uma multiplicação; subtração ao invés de divisão; ou substituição de operações mais complicadas por outras mais simples (por exemplo, $12+12=(10+10)+(2+2) ; 3 \times 7=7+7+7=21$; ou em distúrbios mais sérios: 777$)$. Também é típica a preferência por cálculos escritos de somas (fórmulas) que seriam facilmente calculados silenciosamente, ou calcular contando nos dedos onde a fórmula poderia ser facilmente resolvida silenciosamente ou escrevendo-a sem contar nos dedos (tradução nossa).
} 
Considerando o Gráfico 2, é possível perceber que $46 \%$ dos estudantes apresentaram como hipótese diagnóstica indícios de Discalculia ideognóstica e operacional, representando assim, cinco dos 11 estudantes.

Por meio da análise dos resultados do Subteste de Aritmética (STEIN, 1994), evidenciou-se que é possível que estudantes com indícios de DD apresentem melhoras em seu desempenho quanto à Discalculia ideognóstica e Discalculia operacional, se lhe forem proporcionadas intervenções voltadas as suas dificuldades. As intervenções de acordo com Haase et al. (2011, p. 143), “[...] deve se pautar nas características peculiares de cada indivíduo, de modo a reabilitar seus comprometimentos aritméticos e potencializar as habilidades já apresentadas.".

Contudo, as melhoras no desempenho da Matemática apresentado por esses estudantes, ainda não foram suficientes para descartar a possibilidade da DD. Haase et al. (2011), destacam que a partir do desempenho obtido e considerando o que os autores denominam de resposta à intervenção - RI, as dificuldades dos estudantes persistirem, é possível ter como diagnóstico TA.

\section{Considerações finais}

Este artigo teve como intuito apresentar os resultados advindos da avaliação psicopedagógica, por meio do Subteste de Aritmética (STEIN, 1994), com o objetivo de "Analisar a evolução do desenvolvimento das habilidades matemáticas, após a realização de intervenções psicopedagógicas. ”.

Foi possível verificar que os estudantes E5, E9, E1, E2 e E10 tiveram como hipótese diagnóstica indícios de Discalculia ideognóstica e Discalculia operacional. Já os estudantes E6 e E3 apresentaram indícios de Discalculia léxica, Discalculia ideognóstica, e Discalculia operacional. Em se tratando dos indícios de Discalculia verbal, Discalculia practognóstica, Discalculia léxica, Discalculia gráfica, Discalculia ideognóstica e Discalculia operacional, E7 teve defasagens nos seis subtipos defendidos por Kosc (1974). Referente aos indícios de Discalculia verbal, Discalculia gráfica, Discalculia ideognóstica e Discalculia operacional, destaca-se E8. O estudante E4 foi o único que teve dificuldades somente quanto aos indícios de Discalculia ideognóstica. E o estudante E11 demonstrou defasagens que indicaram indícios de Discalculia verbal, Discalculia ideognóstica, Discalculia operacional.

Concluindo, recomenda-se que pesquisadores dediquem-se a pesquisas referentes à DD e suas comorbidades com outros transtornos. Isso se deve ao seu grau de importância com 
vistas a possibilitar a padronização de testes psicopedagógicos capazes de proporcionarem com precisão se as DAM estão relacionadas aos prejuízos de algum transtorno psiquiátrico, ou se as dificuldades são decorrentes da DD, propiciando assim, que os profissionais realizem intervenções capazes de reabilitarem as habilidades matemáticas em prejuízo.

\section{REFERÊNCIAS}

AVILA, L. A. B. Avaliação e intervenções psicopedagógicas em crianças com indícios de Discalculia. Porto Alegre, 2017. Dissertação (Mestrado) - Pontifícia Universidade Católica do Rio Grande do Sul, 2017.

AVILA, L. A. B.; LARA, I. C. M. Discalculia: Um mapeamento de artigos brasileiros. Abakós. Minas Gerais, v.6, p. 35-56, 2017.

DSM-5. Manual diagnóstico e estatístico de transtornos mentais. 5. ed. Porto Alegre: Artes Médicas, 2014.

FONSECA, V. Abordagem psicopedagógica das dificuldades de aprendizagem. 2. ed. Lisboa: Âncora, 2000.

GIL, A. C. Como elaborar projetos de pesquisa. 5. ed. São Paulo: Atlas, 2002.

HAASE, V. G.; MOURA, R. J.; CHAGAS, P. P.; WOOD, G. Discalculia e Dislexia: Semelhanças Epidemiológica e Diversidade de Mecanismos Neurocognitivos. In: ALVES, L. M; MOUSINHO, R.; CAPELLINI, S. A. (Orgs). Dislexia: Novos temas, novas perspectivas, Publisher: Rio de Janeiro: Wak, 2011, p. 257-282.

HAASE, V. G.; COSTA, D. S.; MICHELLI, L. R.; OLIVEIRA, L. F. S.; WOOD, G. O estatuto nosológico da discalculia do desenvolvimento. In: CAPOVILLA, F. C. (Org). Transtornos de aprendizagem 2: Da análise laboratorial e da reabilitação clínica para as políticas públicas de prevenção pela via da educação, Memnon Edições Científicas: São Paulo, 2011, p. 139-144.

ISLEY, P. Organização Mundial de Saúde - Classificação Estatística Internacional de Doenças e Problemas Relacionados à Saúde. 2015. Disponível em: http://www.datasus.gov.br/cid10/V2008/cid10.htm. Acesso em 19 de maio de 2015.

KOSC, L. Developmentol Dyscalculia. Journal of Learning Disabilities can be found at, v. 7, n. 3, p.163-177, marc. 1974.

KNIJNIK, L. F.; GIACOMONI, C.; STEIN, L. M. Teste de Desempenho Escolar: um estudo de levantamento. Psico-USF, vol. 18, n. 3, Itatiba, p. 407-416, set./dez. 2013.

LARA, I. C. M. Ensino inadequado de Matemática. Revista Ciências e Letras, n. 35, p. 137152, mar./jul. 2004. 
MOOJEN, S.; COSTA, A. C. Semiologia psicopedagógica. In: ROTTA, N. T.;

OHLWEILER, L., RIESCO, R. S. (Orgs). Transtornos da aprendizagem: Abordagem neurobiológica e multidisciplinar. São Paulo: Artes Médicas, 2006. p. 103-112.

MORAES, R. Análise de Conteúdo: limites e possibilidades. In: ENGERS, M. E. A. (Org). Paradigmas e metodologias de pesquisa em educação. Porto Alegre, EDIPUCRS, 1994.

MORAES, R. Análise de Conteúdo. Educação-PUCRS, Porto Alegre, ano XXII (37): 7-32, mar. 1999.

OHLWEILER, L. Introdução. In: ROTTA, N. T.; OHLWEILER, L., RIESCO, R. S. (Orgs). Transtornos da aprendizagem: Abordagem neurobiológica e multidisciplinar. São Paulo: Artes Médicas, 2006. p. 127-130.

RELVAS, M. P. Neurociências e transtornos de aprendizagem: as múltiplas eficiências para uma Educação Inclusiva. Rio de Janeiro: Wak, 2015.

STEIN, L. M. TDE: Teste de Desempenho Escolar: Manual para aplicação e interpretação. São Paulo: Casa do Psicólogo, 1994.

VIEIRA, E. Transtornos na aprendizagem da matemática: Número e discalculia. Revista Ciências e Letras, n. 35, p. 109-120, mar./jul. 2004.

\section{SOBRE AS AUTORAS:}

\section{Lanúzia Almeida Brum Avila}

Doutoranda em Educação em Ciências e Matemática pela PUCRS. Pontifícia Universidade Católica do Rio Grande do Sul - Brasil; Programa de Pós-Graduação em Educação em Ciências e Matemática; Pesquisadora no GEPED/PUCRS. Bolsista CAPES. E-mail: lanuzia.avila@acad.pucrs.br (iD) https://orcid.org/0000-0002-8491-5528

\section{Isabel Cristina Machado de Lara}

Pós-Doutora em Educação em Ciências e Matemática pela PUCRS. Pontifícia Universidade Católica do Rio Grande do Sul - Brasil; Programa de Pós-Graduação em Educação em Ciências e Matemática; Coordenadora do GEPED/PUCRS. E-mail: isabel.lara@pucrs.br

\section{iD https://orcid.org/0000-0002-0574-8590}

